Meta

Journal des traducteurs

Translators' Journal

\title{
Les problèmes de la terminologie aphasiologique
}

\section{Gonia Jarema}

Volume 29, numéro 1, mars 1984

Cerveau, langage et traduction

URI : https://id.erudit.org/iderudit/003256ar

DOI : https://doi.org/10.7202/003256ar

Aller au sommaire du numéro

Éditeur(s)

Les Presses de l'Université de Montréal

ISSN

0026-0452 (imprimé)

1492-1421 (numérique)

Découvrir la revue

Citer cet article

Jarema, G. (1984). Les problèmes de la terminologie aphasiologique. Meta,

29(1), 99-109. https://doi.org/10.7202/003256ar d'utilisation que vous pouvez consulter en ligne.

https://apropos.erudit.org/fr/usagers/politique-dutilisation/ 


\section{LES PROBLÈMES DE LA TERMINOLOGIE APHASIOLOGIQUE}

GONIA JAREMA

Dans le Dictionnaire de la linguistique (Mounin 1974), Claire Maury définit la neurolinguistique de la manière suivante « Domaine de recherche commun à la linguistique et à la neurologie, la neurolinguistique est essentiellement aujourd'hui l'étude de la pathologie du langage, des aphasies. "1 C'est donc de la terminologie aphasiologique qu'il sera question dans la présente étude. Terminologie complexe et parfois hétérogène car, d'une part, elle appartient à un domaine interdisciplinaire, d'autre part, elle reflète, dans une perspective diachronique, l'évolution, voire même les conceptions antagonistes d'une science qui n'a encore cessé, 1) d'interpréter les observations cliniques, 2) d'élaborer une nomenclature qui se veut le juste reflet des phénomènes observés, 3) d'échafauder des taxonomies permettant de classer les groupes symptomatologiques en tableaux cliniques cohérents et 4) d'associer les différents types d'aphasie aux sites des lésions responsables des troubles. On constate par conséquent que la terminologie aphasiologique se fonde essentiellement sur des préoccupations diagnostiques et localisationnelles. Toutefois, ce n'est pas tant l'expression d'un trait sémiologique ${ }^{2}$ (vocabulaire linguistique, psycholinguistique ou physiologique) ni celle des zones anatomiques concernées (vocabulaire médical) qui provoque l'embarras du lecteur, c'est plutôt la pléthore des termes constituant les taxonomies des aphasies. C'est en effet le souci de classer les symptômes aphasiques dans un double objectif diagnostique et thérapeutique qui a donné lieu à des variations terminologiques importantes - variations qui sont loin de faciliter l'identification des syndromes chez les différents auteurs. La littérature aphasiologique foisonne en effet de termes renvoyant à une même entité clinique.

La synonymie, phénomène linguistique naturel, contribue comme tout le monde le sait à l'enrichissement de la langue commune ; en langue de spécialité cependant, elle est à éviter, car elle contrecarre la relation d'univocité qui relie le signifié, ou notion, au signifiant, ou dénomination. L'institutionnalisation de la normalisation terminologique fait preuve du souci d'épuration de la langue de spécialité où l'on aimerait, du moins en théorie, voir un seul terme correspondre à un seul concept bien défini. L'usage est toutefois un facteur tenace, surtout si, comme c'est le cas en aphasiologie, la terminologie reflète l'évolution d'une science et ses différentes approches. Les taxonomies multiples des aphasies rendent ainsi compte du canevas conceptuel de leurs auteurs.

1. Il est significatif à cet égard que Lecours, Lhermitte et al. (1979) proposent une définition partielle commune de l'aphasiologie et de la neurolinguistique : "étude de l'ensemble des corrélations établies entre les caractéristiques cliniques, anatomopathologiques et physiopathologiques des maladies acquises de la parole et du langage, d'une part, et leurs caractéristiques purement linguistiques, d'autre part ", car "Bien qu'elle vise, en définitive, la compréhension des phénomènes biologiques qui sous-tendent les comportements linguistiques normaux, la neurolinguistique est née de l'étude des maladies de la parole et du langage et s'y cantonne encore très largement. "

2. À l'exception de la confusion engendrée par le manque de rigueur étymologique ( $c f$. infra). 
Pour illustrer la confusion qu'engendrent les nomenclatures issues de la démarche onomasiologique des cliniciens, nous allons passer en revue les étiquettes proposées pour les deux "grands " types d'aphasie communément connus sous les noms d'aphasie de Broca et d'aphasie de Wernicke ${ }^{3}$.

\section{APHASIE DE BROCA}

Paul Broca (1861), immortalisé par l'entité clinique qui porte son nom, a dénommé cette dernière aphémie (de $\alpha$-, préfixe privatif et ( $\phi \eta \mu \eta$, parole), terme d'origine grecque dans la tradition onomasiologique médicale, à valeur interprétative. Mais ce terme, considéré, à tort, comme un synonyme étymologique d'infamie, fut bientôt abandonné. Il ressuscite toutefois en 1914, dans la taxonomie de Dejenine, mais pour désigner un tableau clinique distinct, celui de l'anarthrie pure ( $c f$. infra). On le retrouve dans ce même sens chez Goodglass et Kaplan (1972).

Wernicke (1874) appelle l'aphasie de Broca aphasie motrice (perte des images motrices) passant du registre linguistique au registre physiologique. En 1866, ce même auteur, suite aux travaux de Lichtheim (1885) y ajoute des considérations anatomiques en proposant le terme aphasie motrice corticale (terme repris par Goldstein en 1948). Wernicke sous-catégorise en fait l'aphasie motrice en trois types : l'aphasie motrice corticale, l'aphasie motrice sous-corticale et l'aphasie motrice transcorticale - subdivision qu'imposent des profils cliniques à traits séméiologiques distincts.

Pierre Marie (1906) ne prête pas à l'aphasie de Broca un statut indépendant, elle ne porte, par conséquent, aucun nom qui lui soit propre, si ce n'est d'être une aphasie de Wernicke (la seule aphasie reconnue par cet auteur) accompagnée d'une anarthrie. Foix (1928, Pierre et Foix 1917), disciple de Marie, demeure fidèle à l'équation de son mâ̂tre : aphasie de Broca $=$ aphasie de Wernicke + anarthrie.

Pick (1913) et Dejerine (1914) font intervenir, dans leur étiquette aphasie d'expression (terme réutilisé par Weisenburg et Mcbride en 1935), la grande dichotomie troubles de l'expression du langage/troubles de la compréhension du langage - dichotomie qui n'est que grossièrement opératoire, car l'aphasie de Broca peut présenter un trouble de la compréhension et l'aphasie de Wernicke est aussi un trouble de l'expression. En réalité, tout aphasique a un trouble d'expression, ce qui rend l'appellation d'aphasie d'expression, dont la popularité s'est maintenue jusqu'à nos jours, d'autant moins heureuse. Pick emploie aussi le terme du registre anatomique d'aphasie frontale. Head (1926) choisit le terme aphassie verbale (verbal aphasia) qui est encore moins aisé à interpréter et qui n'a guère été retenu. Wepman (1951) utilise l'expression aphasie syntaxique (syntactic aphasia). Luria (1964), s'inscrivant dans la tradition d'une typologie à base physiologique, propose le terme aphasie motrice efférente ${ }^{4}$ ou aphasie motrice cinétique $^{5}$. Jakobson (1956 et 1964) traite les aphasies d'un point de vue strictement linguistique. Il sous-catégorise d'abord les troubles du langage en trouble de la contiguité (contiguity disorder - qui constitue l'aphasie de Broca et qui est un trouble de la combinaison des unités linguistiques - et trouble de la similarité. Puis, influencé par les travaux de Luria, il propose en 1964 une typologie nouvelle plus élaborée, dont la désintégration aphasique par trouble de l'encodage d'unités produites successivement ${ }^{6}$

3. Voir, en annexe, les noms et les définitions des principales formes cliniques de l'aphasie dans la classification de Lecours, Lhermitte et al. (1979).

4. Efférent : qui est issu d'un organe considéré et s'en éloigne (vaisseau, nerf efférent). (Dictionnaire de médecine Flammarion, 1975.)

5. Du grec $\kappa \iota \eta \eta \mu \alpha$ : mouvement.

6. Cette expression, proposée par Lecours, Lhermitte et al. (1979), représente la reconstitution de la description que fait Jakobson de l'aphasie efférente de Luria. Ibid., p. 8. 
qui correspond à l'aphasie de Broca. L'expression trouble de l'encodage (encoding disorder) à caractère nettement psycholinguistique avait déjà été employé par Osgood (1963).

Le degré de fluidité ou fluence (terme calqué sur l'anglais fluency) du discours aphasique, trait séméiologique dont la pertinence clinique est désormais universellement reconnue, a donné lieu à l'appellation aphasie non fluente (non fluent aphasia) ou aphasie du type A (Type A aphasia) chez Howes et Geschwind (1964).

Alajouanine (1968) évoque l'aphasie de Broca sous la rubrique des aphasies de Broca ou aphasies avec troubles de l'articulation verbale, rubrique permettant de distinguer l'aphasie de Broca proprement dite de la grande aphasie de Broca (ou aphasie globale, qui s'installe d'emblée et qui peut évoluer vers une aphasie moins sévère). Le pluriel dont use Alajouanine est repris par Hécaen (1972) qui regroupe sous la rubrique de aphasies d'expression, l'aphasie de réalisation phonématique (aphasie de Broca avec prédominance de troubles arthriques) et l'aphasie agrammatique (aphasie de Broca avec prédominance de troubles syntaxiques).

Mais nombreux sont les auteurs, dont Goodglass et Kaplan (1972) et Lecours, Lhermitte, et al. (1979) qui préconisent l'utilisation d'un terme neutre qui ne préjuge d'aucune façon de la notion véhiculée et préferent ainsi l'éponyme aphasie de Broca. C'est donc le terme qui doit figurer en vedette de la série d'étiquettes proposées pour le syndrome qu'elles identifient, malgré l'emploi fréquent d'aphasie motrice et d'aphasie d'expression.

Les appellations multiples de l'aphasie de Broca donne ainsi lieu au répertoire suivant :

aphasie de Broca

aphémie

aphasie motrice

aphasie motrice corticale

aphasie frontale

aphasie d'expression

aphasie verbale

aphasie syntaxique

aphasie motrice efférente

aphasie motrice cinétique

trouble de la contiguïté

désintégration aphasique par trouble de l'encodage des unités produites successivement

trouble de l'encodage

aphasie de réalisation phonématique

aphasie non fluente

aphasie du type $A$.

\section{APHASIE DE WERNICKE}

Dans une première taxonomie, Wernicke (1874) dégage un type important d'aphasie qui portera plus tard son nom, l'aphasie sensorielle (perte des images acoustiques). On constate, en se rappelant du terme d'aphasie motrice utilisé par ce mêeme auteur pour désigner l'aphasie de Broca, l'homogénéité du registre choisi, Dans sa seconde taxonomie (1885) Wernicke avance une sous-catégorisation de l'aphasie sensorielle identique à celle proposée pour l'aphasie motrice, l'aphasie de Wernicke devient alors l'aphasie sensorielle corticale (terme repris par Goldstein en 1948). 
Selon Marie (1906), il n'existe qu'une seule forme d'aphasie, l'aphasie de Wernicke qui devient donc pour lui l'aphasie. Foix (1928, Marie et Foix 1917) appelle cette dernière la grande aphasie de Wernicke, et dégage un sous-type d'aphasie de Wernicke, avec prédominance des troubles du langage oral, l'aphasie temporale, terme à base anatomique également employé par Pick.

Dejerine (1914) oppose à l'aphasie d'expression (cf. supra) l'aphasie de compréhension (l'aphasie de Wernicke), dichotomie dont nous avons déjà souligné l'inefficacité relative. Son précurseur Pick (1913) avait déjà décrit l'aphasie d'impression.

Le terme aphasie syntaxique (syntactic aphasia), proposé par Head (1926), est un parfait exemple de la non-correspondance entre l'entité clinique décrite et son étiquette. En effet, le syndrome n'est point caractérisé par des troubles syntaxiques et l'appellation de Head est trompeuse, d'autant plus qu'aphasie syntaxique évoque plutôt le tableau clinique de l'aphasie de Broca et sera retenu par Wepman dans ce sens (cf. supra). Wepman (1951) dénomme l'aphasie de Wernicke aphasie pragmatique (pragmatic aphasia).

Jakobson (1956) oppose au trouble de la contiguïté (cf. supra) le trouble de la similarité (similarity disorder) qui constitue l'aphasie de Wernicke et qui est un trouble de la sélection des unités linguistiques. En 1964, il fait intervenir deux nouvelles dichotomies : désintégration vs limitation et encodage vs décodage, ce qui l'amène à décrire la désintégration aphasique par trouble du décodage d'unités produites simultanément ${ }^{7}$. Osgood (1963) utilise lui aussi le terme trouble de décodage (decoding disorder).

Alajouanine (1968) emploie le pluriel, comme pour les aphasies de Broca, et les aphasies de Wernicke ou aphasies sans troubles de l'articulation verbale constituent une famille dont l'aphasie temporale à prédominance sensorielle (l'aphasie de Wernicke) est un sous-type. Mais ces aphasies de Wernicke regroupent aussi certains tableaux cliniques auxquels de nombreux auteurs réservent un statut indépendant. Hécaen (1972) décrit les aphasies sensorielles dont l'aphasie sensorielle avec prédominance de surdité verbale qui correspond à l'aphasie de Wernicke proprement dite.

Si l'éponyme aphasie de Wernicke domine tout comme aphasie de Broca dans la littérature aphasiologique actuelle (Benson et Geschwind 1971; Goodglass et Kaplan 1972 ; Kertesz 1979 ; Lecours, Lhermitte et al. 1979), la pertinence de l'opposition versant auditif/versant visuel contribue, chez Lecours, Lhermitte et al. (1979), au maintien d'au moins deux sous-types d'aphasie de Wernicke : l'aphasie de Wernicke du type I, ou aphasie de Wernicke proprement dite, et l'aphasie de Wernicke du type III, ou aphasie de Wernicke avec prédominance des troubles du langage écrit. Cette dernière est surtout connue sous le nom d'alexie-agraphie (Hécaen 1972) ou d'alexie avec agraphie (alexia with aphasia, Goodglass et Kaplan 1972). On trouve également un terme appartenant au registre anatomique : syndrome pariéto-pli courbe (Alajouanine 1968) ou aphasie par lésion de la région du pli courbe (Marie et Foix 1917). L'aphasie de Wernicke du type II de Lecours, Lhermitte et al. est mieux connue sous le nom d'aphasie sensorielle transcorticale ou de syndrome d'isolement de l'aire du langage (isolation of the speech area syndrome), Geschwind et al. 1968).

Nous pouvons donc établir la liste des diverses appellations de l'aphasie de Wernicke suivante :

aphasie de Wernicke

aphasie sensorielle

aphasie sensorielle corticale

aphasie temporale

aphasie d'impression

7. Cf. note précédente 


\author{
aphasie de compréhension \\ aphasie syntaxique \\ aphasie pragmatique \\ trouble de la similarité \\ désintégration aphasique par trouble du décodage et de la concurrence \\ trouble du décodage \\ aphasie temporale à prédominance sensorielle \\ aphasie sensorielle avec prédominance de surdité verbale \\ aphasie de Wernicke du type I \\ aphasie de Wernicke proprement dite
}

Nous venons de passer en revue les appellations très variées des deux grands syndromes aphasiques : l'aphasie de Broca et l'aphasie de Wernicke. Ces syndromes sont caractérisés par la complexité des manifestations cliniques - complexité qui a contribué à ces variations terminologiques dans la mesure où certains auteurs isolent tel ou tel trait séméiologique important pour désigner une forme clinique souvent préexistante. Le terme ainsi créé porte la marque de la nouveauté, ce qui rend le recours à la néologie et à la multiplication des étiquettes d'autant plus séduisant. Cependant on s'attendrait à ce qu'un type d'aphasie ne présentant qu'un seul trait dominant - et ce type d'aphasie existe et porte le nom d'aphasie pure - soit connu sous un seul et même terme. Paradoxalement, il n'en est rien. Par exemple, l'anarthrie pure affiche elle aussi une myriade d'appellations. Ainsi, Wernicke (1886) la dénomme aphasie motrice sous-corticale. Bastian (1898) lui donne le nom d'aphémie, terme utilisé, comme nous l'avons vu cidessus, par Broca pour désigner le syndrome qui porte son nom. Geschwind (1965) reprend le terme d'aphémie (aphemia) dans le sens que lui donne Bastian. Liepman (1900) utilise le terme d'apraxie articulatoire, Marie (1906) celui d'anarthrie et Dejerine (1914) celui d'aphasie motrice pure. Kleist (1934) forge le terme de mutisme verbal qui devient le mutisme verbal pur (pure word dumbness) chez Brain (1961), Alajouanine (1939) désigne l'anarthrie pure sous le nom de syndrome de désintégration phonétique et Nielsen (1946) sous celui d'aphasie apraxique (apractic aphasia). Goldstein (1948) l'appelle aphasie motrice périphérique (peripheral motor aphasia) et Bay (1962) dysarthrie corticale (cortical dysarthria). Dans la littérature anglophone on trouve également apraxie verbale (verbal apraxia).

Toutefois, l'anarthrie pure est un phénomène très rare et constitue surtout une forme d'évolution de l'aphasie de Broca. Elle ne survient qu'exceptionnellement d'emblée. Le symptôme en tant que tel, qui peut être associé à d'autres manifestations aphasiques est désigné sous le nom de troubles arthriques ${ }^{8}$ ou de désintégration phonétique.

L'anarthrie pure porte donc les étiquettes suivantes:

anarthrie pure

aphasie motrice sous-corticale

aphémie

apraxie articulatoire

anarthrie

aphasie motrice pure

mutisme verbal

mutisme verbal pur

syndrome de désintégration phonétique

aphasie apraxique

8. Perturbation motrice des organes buccophonatoires. 


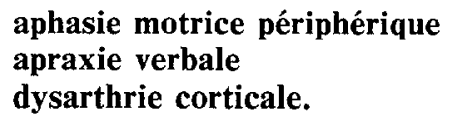

L'étude ponctuelle diachronique des diverses étiquettes apposées aux deux "grands " syndromes aphasiques ainsi qu'à l'une des aphasies dites pures, nous a permis de mettre en relief quelques unes des motivations qui ont contribué à ces variations terminologiques. De toute évidence, les préoccupations séméiologiques l'emportent - elles sont à l'origine des expressions propres au registre linguistique (v.g., trouble de la contiguïté/trouble de la similarité, aphasie syntaxique/aphasie pragmatique), au registre psycholinguistique (v.g., aphasie d'expression/aphasie de compréhension, aphasie d'encodage/aphasie de décodage) ou au registre physiologique (v.g., aphasie motrice (efférente)/aphasie sensorielle). Des critères anatomiques interviennent également, reflétant la volonté d'associer un type d'aphasie particulier à un siège lésionnel spécifique (v.g., aphasie motrice corticale/aphasie sensorielle corticale, aphasie frontale/aphasie temporale, syndrome pariéto-pli courbe). Les auteurs puisent ainsi dans les vocabulaires linguistique, psycholinguistique, physiologique ou anatomique pour forger les termes aphasiologiques - c'est là l'origine des nombreux cas de synonymie observés dans la littérature. C'est aussi ce qui explique l'hétérogénéité qui existe parfois dans la terminologie d'une seule et même taxonomie où se côtoient des termes appartenant à des registres différents. De plus, il faut aussi évoquer la variabilité des dichotomies séméiologiques jugées pertinentes par les auteurs. C'est elle qui donne lieu aux variations quantitatives et qualitatives des symptômes retenus et, par conséquent, des étiquettes qui leur sont apposées. On sait que la création terminologique et la position scientifique de chaque chercheur sont intimement liées. Le recours à la néologie correspond alors soit à la redéfinition d'un ancien concept - et on rebaptise une notion existante ( $v$.g., aphasie motrice $\rightarrow$ aphasie d'expression, aphasie sensorielle $\rightarrow$ aphasie de compréhension), soit à l'identification d'un nouveau concept (v.g., alexie-agraphie). Dans le premier cas, l'auteur risque d'ajouter à la confusion, surtout si le terme est par surcroît sémantiquement trompeur (v.g., aphasie syntaxique pour désigner l'aphasie de Wernicke). Dans le second cas, on aboutit parfois à une sous-catégorisation à outrance, cliniquement peu opératoire. Mais au fur et à mesure que certains termes viennent à refléter des théories abandonnées ou des concepts erronés, une terminologie tend à se décanter pour ne conserver que les termes qui correspondent aux hypothèses courantes. La pléthore embarrassante des étiquettes peut ainsi être considérée comme un problème bibliographique plutôt que pratique. C'est du moins vrai dans le cadre d'une même école où le précepte "un seul terme pour une seule notion " est en général respecté. De plus, les échanges scientifiques internationaux appellent un effort de normalisation. Cependant, les différences linguistiques se fondent, outre sur les différences géographiques et théoriques, sur des traditions scientifiques aux racines socio-culturelles propres, ce qui favorise l'isolationnisme scientifique et terminologique, en dépit de l'accessibilité désormais immédiate de l'information.

$\mathrm{Si}$, dans le présent travail, nous avons surtout examiné le problème de la synonymie en terminologie aphasiologique, c'est que celle-ci constitue le problème le plus saillant de ce domaine. L'homonymie est rare et les quelques cas relevés en diachronie n'ont guère été retenus dans les taxonomies (v.g., le terme d'aphasie syntaxique utilisé pour désigner l'aphasie de Wernicke par Head (1926) et l'aphasie de Broca par Wepman (1961). Le calque, au contraire, est fréquent. L'on se souviendra que les classiques de la littérature aphasiologique sont germanophones, francophones et anglophones. Ainsi, la Leitungs-aphasie de Wernicke devient l'aphasie de conduction et conduction aphasia. 
L'emprunt des notions et la traduction littérale des dénominations d'origine sont facilités par l'isomorphisme des langues mises en jeu (v.g., aphasie motrice/motor aphasia, aphasie sensorielle/sensory aphasia - isomorphisme d'autant plus prononcé qu'il découle souvent d'un fond linguistique commun : les racines et les affixes grecs. C'est à ce propos qu'il faut évoquer le caractère trompeur de certains termes d'origine grecque. Ainsi anomie ( $\alpha$-, privatif et $\nu \sigma \mu o$, loi) devrait se dire d'un trouble structural et non d'un manque du mot, comme c'est le cas. Aussi, à l'encontre de certains aphasiologiques américains (Benson et Geschwind 1971; Goodglass et Kaplan 1972) qui utilisent le terme d'anomic aphasia pour désigner un type d'aphasie caractérisé essentiellement par un manque du mot, les auteurs français préfèrent le terme plus courant d'aphasie amnésique (ou amméstique). Le symptôme en tant que tel, commun à toutes les formes d'aphasie est le manque du mot, beaucoup plus fréquemment qu'anomie, alors qu'anomia prédomine dans la littérature américaine.

Deux préfixes d'origine grecques, a- et dys-, ne cessent de provoquer la confusion. $L a$ parenté sémantique de ces deux préfixes ( $\alpha$ - : privatif, donc absence de et $\delta v \sigma-$ : difficulté) présente sans doute un piège dans la mesure où il ne s'agit pas d'une opposition absolue (v.g., prosodie/aprosodie) mais d'une gradation. Ainsi on a l'aprosodie qui évoque l'absence de traits prosodiques et la dysprosodie qui évoque une perturbation moins sévère, soit une réduction des traits prosodiques. En réalité, l'usage a neutralisé cette nuance et ces termes peuvent s'employer l'un pour l'autre.

En outre, on s'aperçoit en étudiant la terminologie aphasiologique, que les auteurs ne retiennent pas nécessairement l'opposition de degré inhérente à la préfixation en a- et dys-, mais font fi des considérations étymologiques pour mettre de l'avant des distinctions d'un tout autre ordre. Ainsi a- et dys- signalent une opposition entre une aphasie acquise et une aphasie d'évolution dans aphasie (troubles acquis du langage résultant d'une lésion cérébrale) et dysphasie d'évolution (troubles d'acquisition du langage chez l'enfant). Par analogie, ce même sens se retrouve dans les paires :

alexie / dyslexie (troubles de la lecture)

agraphie / dysgraphie (troubles de l'écriture)

acalculie / dyscalculie (troubles du calcul)

Une tout autre opposition est véhiculée par la paire apraxie/dyspraxie. Le premier terme désigne toute perturbation de l'exécution de certains gestes (v.g., tirer la langue, siffler, gonfler les joues, etc., dans l'apraxie buccofaciale) ; le second, une perturbation de l'exécution volontaire des mouvements nécessaires à la production de la parole. Les gestes non langagiers (essentiellement ceux de la face et de la bouche ou ceux des membres) s'opposent ici aux gestes élocutoires.

Autre glissement de sens, l'anarthrie s'oppose à la dysarthrie sur le plan des considérations localisationnelles : l'anarthrie désigne une désintégration phonétique suite à une lésion hémisphérique et la dysarthrie, une désintégration phonétique suite à une lésion du tronc cérébral. Mais les choses se compliquent car dans la littérature aphasiologique de langue anglaise, le terme de dysarthrie (dysarthria) désigne parfois l'anarthrie des auteurs français et celui d'anarthrie (anarthria) une forme très sévère d'anarthrie (ou plutôt de dysarthrie, dans la nomenclature considérée!).

Enfin, un dernier exemple illustrant les nombreux emplois des préfixes a- et dysconcerne les troubles syntaxiques chez l'aphasique. On trouve ainsi l'agrammatisme (perturbation syntaxique d'un stade d'évolution de l'aphasie de Broca, caractérisée surtout par la réduction) et la dyssyntaxie (perturbation syntaxique typique de certaines formes de l'aphasie de Wernicke, caractérisée surtout par les substitutions). Le terme attendu de dysgrammatisme n'existe pas, mais celui de paragrammatisme est synonyme de dyssyntaxie. Le sens véhiculé par ce nouveau préfixe ( $\pi \alpha \rho \alpha-$ : à côté, donc erreur de) 
est beaucoup plus juste, car il fait appel à la notion de déviation, très importante en aphasiologie. Il suffit de se rappeler que les unités linguistiques erronées de l'aphasique sont désignées sous le nom de paraphasies, paralexies ou paragraphies.

Pour conclure, il ressort de la présente étude, qu'aucun terme ne pouvant véhiculer la description totale d'un phénomène (un terme n'est pas une définition), chaque auteur choisit la description présentant le plus de cohérence avec le cadre théorique utilisé - à moins d'opter pour l'éponyme plus facile à manipuler et peu compromettant. Ainsi, non seulement le raisonnement de chacun est-il biaisé par le courant scientifique ambiant, mais le pouvoir d'observation lui-même, l'est à son tour. La terminologie échafaudée pour décrire les phénomènes observés l'est en fin de compte aussi. La pluralité terminologique demeure inévitable tant que nos connaissances comporteront une part d'inconnu, ce qui est indéniablement le cas des sciences neurologiques et de l'aphasiologie. Neurologues, neurolinguistes et psychologues multiplient leurs efforts pour résoudre les problèmes d'une science associant les phénomènes pathologiques, fonctionnels et localisationnels du langage. Le savoir n'étant pas figé, la terminologie ne saurait l'être. Il suffit, pour éviter la confusion, que soient bien définies les choses. L'étiquette conservera toujours sa part d'arbitraire.

\section{LEXIQUE FRANÇAIS-ANGLAIS}

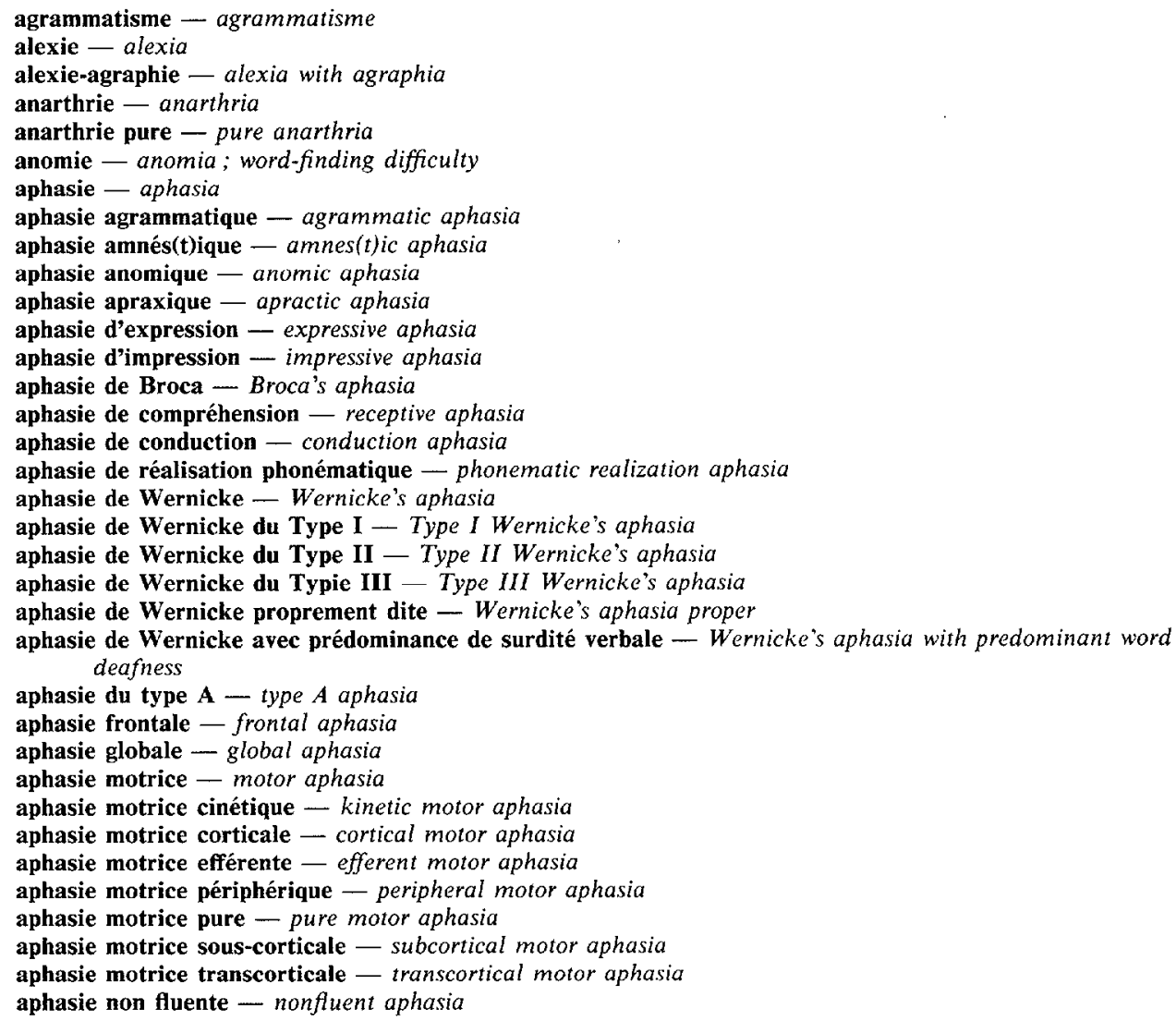


aphasie pragmatique - pragmatic aphasia

aphasie par lésion de la région du pli courbe - aphasia from a lesion in the region of the angular gyrus aphasie sensorielle - sensory aphasia

aphasie sensorielle corticale - cortical sensory aphasia

aphasie syntaxique - syntactic aphasia

aphasie temporale - temporal aphasia

aphasie temporale à prédominance sensorielle - temporal aphasia with sensory predominance

aphasie verbale - verbal aphasia

aphémie - aphemia

apraxie - apraxia

apraxie articulatoire - articulatory apraxia

apraxie verbale - verbal apraxia; apraxia of speech

désintégration phonétique - phonetic disintegration

dysarthrie corticale - cortical dysarthria

dysgraphie d'évolution - developmental dysgraphia

dyslexie d'évolution - developmental dyslexia

dysphasie d'évolution - developmental dysphasia

dyspraxie - dyspraxia

dysprosodie - dysprosody

dyssyntaxie - paragrammatism

grande aphasie de Broca - full Broca's aphasia

grande aphasie de Wernicke - full Wernicke's aphasia

manque du mot - anomia; word finding difficulty

mutisme verbal - word muteness

mutisme verbal pur - pure word dumbness

paragrammatisme - paragrammatism

paragraphie - paragraphia

paralexie - paralexia

paraphasie - paraphasia

syndrome de désintégration phonétique - phonetic disintegration syndrome

syndrome d'isolement (de l'aire du langage) — isolation (of the speech area) syndrome

syndrome pariéto-pli courbe - parieto-angular gyrus syndrome

trouble de l'encodage - encoding disorder

trouble de la contiguïté - contiguity disorder

trouble de la similarité - similarity disorder

trouble du décodage - decoding disorder

troubles arthriques - articulatory impairment

\section{ANNEXE}

classification des aphasies

(Lecours, A.R., F. Lhermitte et al. 1979)

La surdité verbale pure se manifeste par un trouble de la compréhension du langage oral, de la répétition, de l'écriture sous dictée.

La cécité verbale pure se manifeste par un trouble de la compréhension du langage écrit, de la lecture à voix haute, de l'écriture copiée.

L'anarthrie pure se manifeste par des troubles arthriques isolés.

L'agraphie pure se manifeste par l'existence isolée de troubles du graphisme.

L'aphasie de Wernicke proprement dite ou $d u$ Type $I$ : la réalisation arthrique est normale et le discours jargonné; la compréhension et la répétion sont atteintes ; une composante de surdité verbale peut dominer le tableau.

L'aphasie de Wernicke avec prédominance des troubles du langage écrit ou aphasie de Wernicke du Type III : la réalisation arthrique mise à part, le langage est atteint dans tous ses aspects; les troubles de l'expression écrite et l'alexie prédominent toutefois de manière très nette. 
L'aphasie de Broca : en l'absence de troubles immédiatement apparents de la compréhension, ce sont l'anarthrie (les troubles arthriques) et la réduction qui constituent les principaux traits cliniques de l'aphasie de Broca; qu'il s'agisse de l'aphasie de Broca presque limitée aux troubles arthriques ou de la forme commune de l'aphasie de Broca, l'un et l'autre de ces traits cliniques contribuent à masquer la production, pourtant indéniable dès lors qu'il persiste une certaine possibilité d'expression orale et écrite, des transformations paraphasiques et paragraphiques.

L'aphasie de conduction : la compréhension est comparable à celle de l'aphasie de Broca et la réalisation arthrique à celle de l'aphasie de Wernicke; le discours et le langage répété comportent de nombreuses transformations phonémiques (il peut en aller de même de la lecture à voix haute).

L'aphasie amnésique (de Pitres) : le tableau est essentiellement constitué d'un manque du mot se manifestant surtout par la production de périphrases plus ou moins adéquates dans le discours et dans les essais de dénomination.

L'aphasie transcorticale sensorielle ou aphasie de Wernicke du Type II : la réalisation arthrique est normale ; plus ou moins abondant, le discours comporte de nombreuses transformations verbales et le manque du mot y est manifeste; la compréhension du langage est atteinte mais la répétition ne l'est pas; on peut observer une tendance à l'écholalie.

L'aphasie transcorticale motrice (ou syndrome d'aspontanéité) : le trait clinique essentiel est un manque d'incitation verbale se traduisant, dans le discours, par une grande réduction quantitative, il en va de même de l'expression écrite spontanée. La réalisation arthrique est normale ; la dénomination de même que les activités de transposition demeurant possibles.

\section{BIBLIOGRAPHIE}

ALAJOUANINE, Th. (1968) : l'Aphasie et le langage pathologique, Paris, Baillières.

BASTIAN, H. (1898) : Aphasia and Other Speech Defects, London, H.K. Lewis.

BAY, E. (1964) : "Principles of Classification and their Influence on our Concepts of Aphasia", in DeREUCK, A.V.S., M. O'CONNORS (Edits), Disorders of Language, London, Churchill.

BENSON, D.F. et N. GESCHWIND (1977) : "The Aphasias and Related Disturbances", in BAKER, A.B., L.H. BAKER (Edits), Clinical Neurology, vol. 1, New York, Harper and Row.

BRAIN, W.R. (1961) : Speech Disorders, London, Butterworths.

BROCA, P. (1861) : " Remarques sur le siège de la faculté du langage articulé, suivies d'une observation d'aphémie (perte de la parole ", Bulletin de la Société d'anthropologie, 38, Paris, pp. 330-357.

DEJERINE, J. (1914) : Séméiologie des affections du système nerveux, Paris, Masson.

FOIX, C. (1928) : "Aphasies ", in ROGER, G., F. WIDAL et P.J. TEISSIER (édit.), Nouveau traité de médecine, Paris, Masson.

GESCHWIND, N. (1965): "Disconnexion Syndromes in Animals and Man", Brain, 88, pp. 237-294, 585-644.

GESCHWIND, N., F. QUADFASEL et J. SEGARRA (1968) : "Isolation of the speech area", Neuropsychologia, 6, pp. 327-340.

GOLDSTEIN, K. (1948) : Language and Language Disturbances, New York, Grune \& Stratton.

GOODGLASS, H. et E. KAPLAN (1972) : The Assessment of Aphasia and Related Disorders, Philadelphia, Lea and Febiger.

HEAD, H. (1926) : Aphasia and Kindred Disorders of Speech, London, Cambridge University Press.

HECAEN, H. (1972) : Introduction à la neuropsychologie, Paris, Larousse.

HOWES, D. et N. GESCHWIND (1964) : "Quantitative Studies of Aphasic Language", Assoc. Res. Nerv. Ment. Dis., 42, pp. 229-244

JAKOBSON, R. (1956) : "Two Aspects of Language and Two Types of Aphasic Disturbances", in JAKOBSON, R. et M. HALLE (Edits), Fundamentals of Language, The Hague, Mouton.

JAKOBSON, R. (1964) : "Towards a Linguistic Typology of Aphasic Impairments", in DeREUCK, A.V.S et M. O'CONNOR (Edits), Disorders of Language, London, Churchill.

KER TESZ, A. (1979) : Aphasia and Associated Disorders : Taxonomy, Localization, and Recovery, New York, Grune and Stratton. 
KLEIST, K. (1934) : Gehirnpathologie, Barth, Leipzig.

LECOURS, A.R., F. LHERMITTE et al. (1979) : l'Aphasie, Paris, Montréal, Flammarion, PUM.

LICHTHEIM, L. (1885) : "On Aphasia", Brain, 7, pp. 433-484.

LIEPMANN, H. (1900) : Das Krankheitsbild der A praxie (" motorischen Asymbolie "), Monatsschrift für Psychiatrie und Neurologie, Bd. VIII, Monographie, Berlin.

LURIA, A.R. (1964) : "Factors and Forms of Aphasia", in DeREUCK, A.V.S. et M. O'CONNOR (Edits), Disorders of Language, London, Churchill.

MARIE, P. (1906) : " Révision de la question de l'aphasie : la troisième circonvolution frontale ne joue aucun rôle spécial dans la fonction du langage ", La Semaine Médicale, 26, pp. 241-247.

MARIE, P. et C. FOIX (1917) : "Les aphasies de guerre ", in Revue neurologique, 24, pp. 53-87.

MOUNIN, G. (1974) : Dictionnaire de la linguistique, Paris, PUF.

NIELSEN, J.M. (1946) : Agnosia, Apraxia, Aphasia, New York, Hoeber.

OSGOOD, C.E. et M.S. MIRON (Edits) (1963) : Approaches to the Study of Aphasia, Urbana, University Illinois Press.

PICK, A. (1913) : Die Agrammatischen Sprachstörungen, Berlin Springer.

WEISENBURG, T. et K. McBRIDE (1935) : Aphasia, New York, Commonwealth Fund.

WEPMAN, J.M. (1951) : Recovery from Aphasia, New York, Ronald.

WERNICKE, C. (1874) : "Der Aphasische Symptomenkomplex, Cohn et Weigart, Breslau", in Boston Studies on the Philosophy of Science, vol. IV, Dordrecht, Reidel.

WERNICKE, C. (1886) : " Die Neueren Arbeiten über Aphasie ", Fortschritte der Medizin, 4, pp. 371-377. 